\title{
Superficies bioactivas en implantología: una nueva perspectiva
}

\author{
CUTANDO A* \\ GÓIMEZ-MORENO G** \\ ARANA C***
}

Cutando A, Gómez-Moreno G, Arana C. Superficies bioactivas en implantología: una nueva perspectiva. Av Periodon Implantol. 2007; 19, Suppl: 43-50.

\begin{abstract}
RESUIMEN
Actualmente, tras años de investigación en implantología, en los cuales se han realizado muchos trabajos, en donde se han estudiado diferentes tipos de superficies de implantes, lisas y rugosas, en un intento de mejorar, tanto en el tiempo como en la calidad, la osteointegración, se ha concluido que los implantes de superficie rugosa con un intervalo de rugosidad entre 1,0-2,0 nm, son los que parecen dar una mejor respuesta ósea y mejores resultados clínicos, pero desde un punto de vista exclusivamente mecánico. Hoy en día las investigaciones se centran en el desarrollo de superficies denominadas bioactivas, las cuales son capaces de interaccionar con el hueso que rodea al implante, como ocurre cuando el implante es revestido con carbonato cálcico o bien con flúor. Pero sabemos que dentro del metabolismo óseo, juegan importantes papeles, moléculas, como son la melatonina y la hormona del crecimiento entre otras, las cuales deben de ser tenidas en cuenta a la hora de hablar de osteointegración. Se realiza una breve descripción de la fisiología de ambas moléculas y se valora su aplicación al concepto de superficie bioactiva en implantología.
\end{abstract}

\section{PALABRAS CLAVE}

Implante, superficie bioactiva, melatonina, hormona del crecimiento.

Fecha de recepción: Diciembre 2006.

Fecha de aceptación: Enero 2007.

\section{INTRODUCCIÓN}

Las investigaciones actuales en implantología van encaminadas a conseguir una rápida integración del implante con el tejido óseo que le rodea. El éxito o fracaso de este proceso depende del comportamiento de la interfase tejido óseo-implante (1). En este proceso participan muchas variables: Tipo de material utilizado, forma, topografía y tipo de superficie sin olvidar cargas mecánicas, técnica quirúrgica y aspectos inherentes a cada paciente que afectan a su metabolismo óseo, sin olvidarnos tampoco de los tejidos blandos, los cuales participan del éxito del implante formando una barrera alrededor del implante protegiendo la

\footnotetext{
* Profesor Titular en la Unidad Docente de Clínica Odontológica Integrada de Pacientes Especiales. Departamento de Estomatología. Facultad de Odontología. Universidad de Granada. Granada. España.

** Profesor Asociado en la Unidad Docente de Clínica Odontológica Integrada de Pacientes Especiales. Departamento de Estomatología. Facultad de Odontología. Universidad de Granada. Granada. España.

*** Doctorando en el Departamento de Fisiologia. Instituto de Biotecnologia. Universidad de Granada. Granada. España.
} 
interfase implante-hueso (2). Cuando todo el proceso tiene éxito hablamos de osteointegración, situación que se define como: "El hueso esta en directo contacto con el implante sin ningún tejido conectivo en medio " (3). Este mismo autor posteriormente matiza su definición de osteointegración: "Es una conexión directa tanto estructural como funcional, entre el hueso vivo y la superficie de un implante preparado para soportar una carga" (4). No existe por lo tanto ningún tipo de movimiento entre el implante y el hueso que le rodea, ósea hay una osteointegración física o mecánica. Esta integración mecánica le da al implante una buena resistencia frente a las fuerzas de tracción, pero una baja resistencia a las fuerzas de tensión, las cuales se producen perpendiculares al hueso que rodea al implante (5).

Hay autores que defienden la presencia de interacciones de tipo químico entre el implante y el hueso que le rodea. Este hecho aparece al aplicar a la superficie del implante materiales reactivos del tipo, fosfato cálcico y flúor, que son capaces de conseguir uniones químicas entre el implante y el hueso que le rodea. Este concepto a dado lugar al término de óseocoalescencia como sinónimo de fundirse el implante y el hueso. Esta forma de integración parece que tiene una buena resistencia tanto a las fuerzas de tracción, como a las de tensión (5).

Por lo tanto podemos afirmar que el éxito del implante depende del tipo de superficie de éste, y de la respuesta del hueso que lo rodea. Si nos centramos en la topología de la superficie, se acepta que tiene un importante papel en la diferenciación osteoblástica y en la mineralización ósea (6). Ya aquí, se distinguen implantes de superficie rugosa e implantes de superficie lisa. Estudios in vitro han demostrado que las células osteoblásticas tienden a actuar mas rápidamente ante superficies rugosas, hecho que se manifiesta en un aumento de la diferenciación de estas células óseas, un aumento de la actividad de la fosfatasa alcalina, junto a un aumento en la producción de osteocalcina y de colágeno. Esta respuesta biológica a nivel óseo, esta modulada por diferentes moléculas como son la Vitamina D3, factores de crecimiento local,TGF-B y la PGE2, $(7,8)$. Así, el TGF-B estimula la replicación de líneas celulares precursoras de osteoblastos y de la síntesis de colágeno a nivel del tejido óseo. La prostaglandina a bajas concentraciones, PGE2, impulsa el crecimiento óseo y la síntesis de colágeno tipo I. A altas concentraciones tiene el efecto contrario. La vitamina D3 promueve la expresión y actividad de la fosfatasa alcalina, junto a un aumento en la producción de colágeno tipo I y de la osteocalcina por las células osteoblásticas (9).
A diferencia de los osteoblastos, los fibroblastos se adhieren mas fácilmente a las superficies de los implantes no rugosos o lisos ofreciendo poca resistencia a la tracción (10).

\section{CARACTERÍSTICASS DE LAS SUPERFICIES DE LOS IMPLANTES}

Dos tipos de superficies han sido comúnmente relacionadas con la respuesta del entorno óseo, la forma o topografía, ya sea rugosa o lisa, del implante y la otra superficie hace referencia a las propiedades químicas. $\mathrm{Si}$ bien es cierto que a veces las modificaciones en la topografía del implante conlleva cambios de tipo químico en la misma superficie (11).

A. Superficies lisas. Se obtienen a través de:

- Electropulido. "Electropolished". La superficie del implante es tratada electroquímicamente por inmersión en un baño electrolítico a través del cual pasa una corriente eléctrica.

- Torneado "Machined or turned": La superficie sufre un proceso de torneado mecánico que macroscópicamente pule y alisa la superficie (12).

\section{B. Superficies rugosas. Se obtienen:}

\section{- Añadiendo materiales}

- Técnica Spray plasma-titanio, "Titanium plasma spray, (TPS)". En este método se utiliza un gas noble compuesto por iones, electrones y plasma, los cuales son comprimidos a alta temperatura y lanzados a una velocidad de $3.000 \mathrm{~m} / \mathrm{seg}$. A través de este método se obtiene una rugosidad de aproximadamente $15 \mathrm{~nm}$.

\section{- Quitando materiales}

- Técnica de Sandblasting. En esta técnica la superficie del implante es bombardeada con un chorro de aire y agentes abrasivos, arena, a una presión controlada, aumentando de esta manera la superficie de contacto (13).

- Técnica del grabado ácido y chorreado, "Acid etching and blasting". En esta técnica, la superficie del implante es bombardeada con gruesas partículas de entre 250-500 nm. Dichas partículas son generalmente de Al3O3. Posteriormente, se graba con ácido clorhídrico y con ácido sulfúrico. Este tipo de tratamiento de la superficie parece ser que es en la que mejores resultados de osteointegración se producen (14). 
- Técnica del grabado ácido,"Acid etching". En este proceso se utilizan soluciones ácidas para tratar las superficies de los implantes a través de erosiones químicas.

Siguiendo a Albrektsson (15) podríamos enfocar a las superficies de los implantes no como se fabrican sino en su calidad, característica o propiedades. Así habla de propiedades mecánicas, topográficas y físico-químicas.

- Las propiedades mecánicas, hacen referencia al potencial estrés, fatiga o envejecimiento de la superficie del implante. Esto puede traer como consecuencia la corrosión del implante. Esta propiedad no ha sido suficientemente estudiada (16).

- Las propiedades topográficas. Se refiere al grado de rugosidad de la superficie del implante, así a como están orientadas sus irregularidades. Esta propiedad ha sido el centro de todas las investigaciones realizadas durante la última década en relación a superficies de implantes (17).

- Propiedades físicas. Se refieren a la presencia en la superficie del implante de factores tipo energía y carga. Así una superficie con alta energía tiene una alta capacidad de absorción, y por lo tanto el implante tendría, supuestamente, mas capacidad de osteointegración, en comparación con implantes de mas baja energía (18).

- Propiedades químicas. Este aspecto de las superficies del implante están tomando cada día mas importancia, a través de las múltiples investigaciones que se están realizando. Dependiendo de la composición química, la superficie provoca diferentes reacciones en el hueso que la rodea., con cambio de agua e iones que condicionan la unión de las proteínas y por tanto la reacción celular, las cuales intentan conseguir una unión implante-hueso bioquímica, que permita un buen anclaje del implante. Una definición de este concepto sería aquella que dijese: "Es la característica de un implante que le permite formar una unión con los tejidos vivos"(19). Aunque esta definición no recoge el hecho de que aunque exista un anclaje de tipo químico, también la hay de tipo mecánico, compartiendo ambas propiedades, el éxito del implante. Sin embargo la propiedad bioquímica del implante le permite una unión mucho mas rápida.

Actualmente existen dos tipos de implantes con superficies que se presentan como superficies bioactivas:

1. Implantes con fosfato cálcico. Se basa su actividad en la capacidad de formar apatita impulsando la función celular para conseguir formar una interfase de hueso y carbonato cálcico (20).

2. Implantes con flúor. Los resultados obtenidos con este tipo de superficie con flúor parecen demostrar una buena adhesión del hueso en la interfase en comparación con los controles, demostrando una reacción bioactiva de estos implantes (21).

Después de esta pequeña revisión, podemos sacar la consecuencia de que las superficies moderadamente rugosas comparadas con las superficies mas lisas, poseen ventajas clínicas, y que las superficies de implantes bioactivas parecen representar el futuro (22).

Sin embargo todo estos intentos actualmente pasan por recubrir el implante, cuando hablamos de superficies bioactivas, de minerales, iones, o moléculas similares, no teniendo en cuenta otras moléculas como pueden ser las diferentes hormonas, entre otras moléculas, implicadas en el metabolismo óseo, así como factores de crecimiento óseo, etc, que podrían favorecen una mas rápida y adecuada respuesta ósea. En relación a esto tenemos dos moléculas que en el campo de la medicina se ha valorado muy seriamente su aplicación en determinadas patologías óseas, hablamos de la melatonina, (MLT) y de la hormona de crecimiento (HG).

\section{¿QUÉ ES LA MELATONINA?}

La melatonina (MLT), es la N-acetil-5-metoxitriptamina, una hormona sintetizada y secretada principalmente, por la glándula pineal, descrita inicialmente por McCord y Allen (1917) y aislada por primera vez por Lerner y cols. (1958) $(23,24)$.

La melatonina se libera durante la noche a través de la activación postsináptica de receptores $\beta$-adrenérgicos. Esto es así, ya que la luz evita la activación adrenérgica de la glándula pineal, produciéndose una inhibición en la síntesis de melatonina. Dentro de las variadas funciones de la melatonina está reconocida su acción:

\section{ESTIMULADORA DE LA FORMACIÓN ÓSEA}

Numerosos trabajos señalan a la melatonina como un importante mediador en la estimulación y formación de hueso. A concentraciones micromolares, la melatonina estimula la proliferación y la síntesis de fibras de colágeno tipo I en osteoblastos humanos in vitro (25). 
Por otra parte, en cultivos de preosteoblastos procedentes de ratas, aumenta la expresión génica de la sialoproteína ósea y de otros marcadores proteicos de hueso, incluyendo la fosfatasa alcalina, la osteopontina y la osteocalcina, de una manera dosis-dependiente, reduciendo su periodo de diferenciación a osteoblastos de 21 días, que es lo normal, a 12 días (26).

Dentro del metabolismo óseo, otra importante acción biológica directa de la melatonina se centra en el osteoclasto, célula multinucleada encargada de la reabsorción de matriz extracelular mediante diversos mecanismos, dentro de los cuales se encuentra la producción de radicales libres. La melatonina, a través de su acción antioxidante y depuradora de radicales libres, podría interferir en esta función del osteoclasto e inhibir de esta forma la reabsorción ósea (27). Esta inhibición en la reabsorción ósea se vería potenciada por otra acción de la indolamina a nivel de la osteoclastogénesis, ya que Koyama et al. han observado que la aplicación de melatonina en rangos de 5 a $500 \mu \mathrm{M}$ disminuye de manera dosis-dependiente la expresión de mRNA de RANK y aumenta tanto los niveles de OPG como de mRNA de OPG en líneas celulares preosteoblásticas MC3T3-E ${ }_{1}$ (28). Pero que es esta triada, OPG/ RANKL/RANK?

\section{SISTEMA OPG/RANK/RANKL}

La acción biológica del osteoclasto se ve regulada primordialmente por la tríada molecular OPG/RANK/ RANKL (29). El receptor RANK (receptor activador del factor nuclear $\mathrm{kB}$ ) es un péptido que se expresa en osteoclastos maduros y preosteoclastos, cuya activación da lugar a cambios en el citoesqueleto y actividad del osteoclasto, de tal forma que induce a esta célula a reabsorber hueso y a no morir por apoptosis; se trata de una señal de activación y supervivencia para el osteoclasto. El ligando encargado de activar al receptor RANK es RANK-L (ligando de unión al receptor activador del factor nuclear $\mathrm{kB}$ ), que es una molécula que aparece anclada a la membrana del osteoblasto, aunque aparece también en células inmaduras mesenquimales de médula ósea. Su principal función es, mediante la unión a RANK, estimular la diferenciación de los osteoclastos, su activación y la inhibición de su apoptosis. Existe una tercera molécula, que es la osteoprotegerina (OPG). La cual se llama también factor de inhibición de la osteoclastogénesis y es un nuevo miembro de la superfamilia de los receptores del factor de necrosis tumoral (TNFR) que se expresa en diversos tejidos humanos además de en el hueso. A ni- vel óseo, inhibe la maduración y activación de los osteoclastos al unirse al RANKL e impedir la unión del RANK (30). La melatonina puede bloquear el receptor osteoclástico RANK impidiendo su activación por RANKL y por tanto el inicio de la diferenciación osteoclástica que daría lugar a la reabsorción ósea.

Todos estos datos confirman un efecto osteogénico de la melatonina que puede ser clínicamente importante, ya que podría ser usada como potencial agente terapéutico en situaciones en las que sea deseable un aumento de la formación ósea, como cicatrización de fracturas u osteoporosis, o su uso como, una superficie bioactiva en implantología (31).

\section{¿QUÉ ES LA HORMONA DE CRECIMIENTO (GH)?}

La hormona de crecimiento (GH) es un péptido de una sola cadena con 191 aminoácidos, que es secretado por el adenohipófisis en respuesta a la liberación del factor de crecimiento de la GH, (GHRF), producido por el hipotálamo. La producción de $\mathrm{GH}$ es regulada casi exclusivamente por el sistema nervioso central.

La deficiencia de GH produce enanismo y la sobreproducción causa gigantismo o acromegalia. La GH, estimula la síntesis de proteínas en todas las células, la movilización del tejido adiposo y el uso de ácidos grasos para obtener la energía, reduciendo el uso de hidratos de carbono. Su acción en el crecimiento depende de la presencia de tiroxina, insulina, e hidratos de carbono $(32,33)$. Las somatomedinas, producidas principalmente en el hígado en respuesta al estímulo por $\mathrm{GH}$, promueven el crecimiento esquelético, aunque la GH no sea capaz de promover el alargamiento de los huesos largos una vez que las epífisis están cerradas (34).

La GH es una de las sustancias reguladoras más importantes en el crecimiento de hueso y en el remodelado de hueso vivo (33). Esta, directamente aumenta el crecimiento longitudinal del hueso estimulando los precondrocitos. Parece ser que la GH no sólo tiene efectos endocrinos, sino también efectos paracrinos y autocrinos. En este momento se piensa que, la GH, es un factor de crecimiento local, ya que se acepta de que, aparte de ser producida por lo adenohipófisis, la producen muchas células incluidas los osteoblastos (35).

Sus efectos son mediados principalmente por un péptido de 70 aminoácidos, factor-I de crecimiento pare- 
cido a la insulina, insulin-like growth factor-I, (IGF-I), que es sintetizado en la mayor parte de los tejidos, pero principalmente en el hígado y en el tejido condral ante el estímulo de la GH. Así mismo esta hormona puede inducir la proliferación y la diferenciación de osteoblastos, los cuales tienen receptores para esta hormona, aumentando la incorporación de timidina H3 dentro de dichas células así como de otros marcadores bioquímicos con fenotipo osteogénico como son el procolágeno Tipo I, osteocalcina y fosfatasa alcalina (36).

También estimula la producción de factores de crecimiento locales que son análogos a insulina, IGF-I y IGFII, los cuales estimulan a los osteoblastos remodelando de esta manera el hueso (37). El IGF-I e IGF-II,estos factores aparecen en gran cantidad en la matriz osteoide (38).

El IGF-II, es el factor de crecimiento más abundante en la matriz de hueso, y tiene un importante papel en la embriogénesis. Pero sus efectos a nivel del esqueleto desarrollado son actualmente desconocidos (40).

La GH actúa indirectamente en el hueso a través de la síntesis aumentada de IGF-I e IGF-II por los osteoblastos. Induciendo el crecimiento del hueso a través del estímulo directo que realiza sobre los condrocitos de la epífisis y sobre los osteoblastos (41).

En 1969 el aumento de la masa de hueso fue demostrado por primera vez después de un tratamiento sistémico con GH en perros (42). Así mismo, aumento la densidad mineral ósea, en individuos de más de 60 años después de seis meses del tratamiento con $\mathrm{GH}$ (43). La GH puede estimular el "turnover" del hueso, aumentando el número de factores que participan en la reabsorción de hueso y aquellos que lo hacen en la formación de hueso (44).

La GH sistémica ha sido usada experimentalmente para inducir la reparación en fracturas de hueso tanto en ratas viejas como en jóvenes, presentando un aumento del $400 \%$ de propiedades biomecánicas en comparación con un grupo de control no tratado (45). Recientes estudios han demostrado la acción local de la $\mathrm{GH}$. Este hecho se manifiesta por una intensa remodelación ósea así como la estimulación de la síntesis de colágeno, osteocalcina y fosfatasa alcalina (46).

A la vista de lo expuesto anteriormente, queda claro la acción local que sobre el metabolismo óseo ejercen estas dos moléculas. Efecto positivo sobre la formación de hueso. Estamos investigando sin parar como mejorar nuestra osteointegración implantaria, pero solo lo estamos haciendo sobre un pilar de los dos que tiene este proceso, este pilar es la superficie del implante, pero no estamos haciendo nada sobre el metabolismo óseo que se produce alrededor de dicha superficie. Podemos potenciar dicho metabolismo en la dirección de conseguir una mejora de la producción de matriz osteoide a través de una estimulación de la acción osteoblástica y una disminución de la osteoclastogénesis, como ocurre con estas dos moléculas. Sin lugar a dudas esto repercutirá en una más rápida y mejor osteointegración.

\section{SUMMMARY}

At present, after years of investigation in implantology, in which there have been realized a lot of works, with different types of surfaces of implants, smooth and rough, in an attempt of improving, both in the time and in the quality, the osteointegration, one has concluded that the implants of rough surface with an interval of rugosity between 1.0-2.0 $\mathrm{nm}$, are those who seem to give a better bone response and better clinical results, but from an exclusively mechanical point of view. Nowadays the investigations centre on the development of named bioactive surfaces, which are capable to react with the bone around the implant, since it happens when the implant is redressed in carbonate cálcico or in fluorine. But we know that inside the bone metabolism, there are another important molecules: the melatonin and the hormone of the growth between others, which must be born in mind at the time of speaking of osteointegration. There is realized a brief description of the physiology of both molecules and his application is valued to the concept of bioactive surface in implantology.

\section{AGRADECIMIENTOS}

Proyecto de Investigación: "Estudio del sinergismo entre Melatonina y Hormona de Crecimiento (GH) sobre los procesos de osteointegración en implantes dentales y regeneración ósea en la cavidad oral", financiado por:

- Fondo de Investigación Sanitaria (FIS). Ministerio de Sanidad y Consumo (Ref.: PI041610).

- Ministerio de Educación y Ciencia y Microdent Implant System (Proyecto PETRI n. 95-0885-OP).

- Grupo de Investigación CTS-263 (Junta de Andalucía). 


\section{KEY WORDS}

Implant, bioactive surface, melatonin, hormone of the growth.

\section{RÉSUMÉ}

Actuellement, après des années d'investigation dans implantology, dans lesquels ont été réalisés beaucoup de travaux, dans lesquels ont été étudiés différents types de surfaces d'implants, plates et rugueuses, dans une tentative d'aller mieux, dans le temps et dans la qualité, l'osteointegración, on a fini que les implants de surface rugueuse avec un intervalle de rugosité entre 1,0-2,0 nm, sont ceux qui semblent donner une meilleure réponse osseuse et de meilleurs résultats cliniques, mais d'un point de vue exclusivement mécanique. Aujourd'hui les investigations se concentrent sur le développement de bioactives dénommées surfaces, lesquelles sont capables d'intergesticuler avec l'os qui s'entoure à l'implant, comme il arrive quand l'implant est revêtu par un carbonate calcique ou bien par fluor.

Mais nous savons que dans le métabolisme osseux, ils jouent des papiers importants molécules, comme c'est le melatonin et l'hormone de la croissance entre les autres, lesquelles sont probablement tenues en compte à l'heure de parler d'osteointegración. On réalise une brève description de la physiologie des deux molécules et son application est évaluée au concept de bioactive surface dans implantología.

\section{BIBLIOGRAFÍA}

1. Puleo DA, Thomas MV. Implants surfaces. Dent Clin North Am 2006;50:323-38.

2. GlauserR Schupbach P, Gottlow J, et al. Periimplant soft tissue barrier at experimental one-piece mini-implants with different surface topography in humans: a lightmicroscopic overview and histometric analysis. Clin Implant Dent Relat Res 2005; 7: 44-51.

3. Branemark PI, Hansson BO, Adell R, et al. Osseointegrated implants in the treatment of the edentulus jaw. Experiences from a 10 years period. Scand J Plast Reconstr Surg. 1977; 16:1-132.

4. Branemark PI, Zarb G, Albrektsson T. Tissue-integrated prostheses: Osseointegration in clinical dentistry. Quintessence Publishing Co, Chicago. 1985.
5. Kasemo B, Gold J. Implant surfaces and interface processes. Adv Dent Res. 1999; 13-21.

6. Cooper LF, Masuda T, Whiston SW, Yliheikkila P, Felton DA. Formation of mineralizing osteoblast cultureson machined titanium surface. Int J Maxilllofac Implants. 1999; 14:37-34.

7. Boyan BD, Batzer R, Kieswetter K, Liu Y, Cochran DLK, et al.Titanium surfaces roughness alters responsiveness of MG63 osteoblast-like cells to 1 alfa,25-(OH)2 D3. J Biomed Mater Res 1998;39:77-85.

8. Papparlardo S, Baglio OA, Cappello V, Mortellaro C, Grassi FR. Implant surface and osseointegration. Minerva Stomatol 2005;54:66 1-73

9. Kieswetter K, Schwartz Z, Hummert TW, Cochran DL, Simpson J. Surface roughness modulates the local production of growth factors and cytokines by osteoblastlike (MG63) cells. J Biomed Mater Res 1996;32:55-63.

10. Brunski JB, Puleo DA, Nanci A. Biomaterials and biomechanics of oral and maxillofacial implants: current status and future developments. Int J Oral Maxillofac Implants 2000;15:15-46.

11. Szmukler-Moncler S, Salama H, Reingewirtz Y, et al. Timing of loading and effect of micromotion on bonedental implant interface:review of experimental literature. J Biomed Mater Res 1998;43:192-203.

12. Cordioli G, Majzoub Z, Piatrelli A, scarano A. Removal torque and histomorphometric investigation of 4 diferent titanium surfaces: an experimental study in the rabit tibia. Int J Oral Maxillofac Implants 2000;15:668-14.

13. Piatelli A, Scarano A, Piatelli M, Calabrese L. Direct bone formation on sand-blasted titanium implants:an experimental study. Biomaterials 1996;17:1015-8.

14. Buser D, Belser UC, Lang NP. The original one stage dental implant system an dits clinical applications. Periodontol 200 1998;17:106-18.

15. Albrektsson T. Oral implants surfaces: Partl, review focusing on topographic and chemical properties of different surfaces and in vivo reponses to them. Int J Prosthodont 2004;17:536-43.

16. Kohn DH, Ducheyne P. A parametric study of the factors affecting the fatigue strength of porous-coated Ti6AI4V implant alloy. J Biomed Mater Res 1990;24:1483-501. 
17. Eckert SE, Parein A, Myshin HL, Padilla JL. Validation of dental implant system through a review of literature supplied by system manufactures. J Prosthet Dent 1997;77:271-9.

18. Baier RE. Surface preparation. J Oral Implantol 1986;12: 387-95.

19. Hench LL. Bioactive glasses and glass ceramics: A perspective. In: Yamamuro T, Hench L, Wilson J (eds). Handbook of Bioactive Ceramics Vol I. Boca Raton, FL:CRC, 1990:7-23.

20. Jansen JA, Brugge P, Van Der Waal E, Vredenberg A, Wolke J. Osteocapacities of calcium phosphate ceramics. In: Ellingsen JE, Lyngstadaas SP(eds). Bioimplant Interface. Boca Raton, FL:CRC 2003:305-22.

21. Ellingsen JE. Increasing biocompatibility by chemical modification of titanium surfaces. In: Ellingsen JE, Lyngstadaas SP(eds) Bioimplant Interface. Boca raton, FL:CRC; 2003:323-40.

22. Jokstad A, Brägger U, Brunski J, Carr AB, Naert I, Wennerberg A. Quality of dental implants. Int Dent J. 2003;53:409-43.

23. McCord CP, Allen FB. Evidences associating pineal gland function with alterations in pigmentation. J Exp Zool 1917; 23:207-24.

24. Lerner $\mathrm{AB}$, Case JD, TakahashiY, Lee TH, Mori W. Isolation of melatonin, the pineal gland factor that lightens melanocytes. J Am Chem Soc 1958; 80:2587.

25. Nakade O, Koyama H, Ariji H,Yajima A, Kaku T. Melatonin stimulates proliferation and type I collagen synthesis in human bone cells in vitro. J Pineal Res 1999; 27:10610.

26. Roth JA, Kim BG, Lin WL, Cho MI. Melatonin promotes osteoblast differentiation and bone formation. J Biol Chem 1999; 274:22041-7.

27. Cardinali DP, Ladizesky MG, Boggio V, Cutrera RA, Mautalen C. Melatonin effects on bone: experimental facts and clinical perspectives. J Pineal Res 2003;34:81-7.

28. Koyama H, Nakade O, Takada Y, Kaku T, Lau KH. Melatonin at pharmacologic doses increases bone mass by suppressing resorption through down-regulation of the RANKL-mediated osteoclast formation and activation. J Bone Miner Res 2002;17:1219-29.
29. Riancho JA, Gutiérrez GE. Factores Reguladores de la Resorción Ósea. Rev Metab Oseo Min 2003; 1(2):51-66.

30. Fernández-Tresguerres Hernández-Gil I, Alobera García MA, Del Canto Pingarrón M, Blanco Jerez L. Bases fisiológicas de la regeneración ósea II. El proceso de remodelado. Med Oral Patol Oral Cir Bucal 2006;1 1:151-7.

31. Cardinali DP, Ladizesky MG, Boggio V, Cutrera RA, Mautalen C. Melatonin effects on bone: experimental facts and clinical perspectives. J Pineal Res 2003;34:81-7.

32. Ohlsson C \& Vidal O. Effects of growth hormone and insulin-like growth factors on human osteoblasts. European Journal of Clinical Investigation 1998; 28:184-6.

33. Ohlsson C, Bengtsson BA, Isaksson OG, Andreassen TT \& Slootweg MC. Growth hormone and bone. Endocrine Reviews 1998;19:55-79.

34. Harris WH \& Heaney RP. Effect of growth hormone on skeletal mass in adult dogs. Nature 1969;223:403-4.

35. Harvey S \& Kull KL. Growth hormone, a paracrine growth factor? Endocrine 1998;7:267-79.

36. Kassem M, Blum W, Ristelli J, Mosekilde L. \& Eriksen EF. Growth hormone stimulates proliferation and differentiation of normal human osteoblast-like cells in vitro. Calcified Tissue International 1993; 52:222-6.

37. Chenu C, Valentin-Opran A, Chavassieux P, Saez S, Meunier PJ \& Delmas,PD. Insulin-like growth factor I hormonal regulation by growth hormone and by 1,25 $(\mathrm{OH})_{2} \mathrm{D}_{3}$ and activity on human osteoblast-like cells in short-term cultures. Bone1990;11:81-6.

38. Cohick WS. \& Clemmons DR. The insulin-like growth factors. Annual Review of Physiology 1993; 55:131-53.

39. Hill PA, Reynolds JJ \& Meikle MC. Osteoblasts mediate insulin-like gowth factor-I and -II stimulation of osteoclasts formation and function. Endocrinology 1995; 136 : 124-31.

40. Mohan S \& Baylink DJ. Bone growth factors. Clinical Orthopaedics and Related Research 1991;263:30-48.

41. Mosekilde L, Thomsen JS, Orhii PB \& Kalu DN. Growth hormone increases vertebral and femoral bone strength in osteopenic, ovariectomized, aged rats in a dosedependent and site-specific manner. Bone 1998;23:34352 . 


\section{AVANCES}

Volumen 19 - Suppl. - Febrero 2007

42. Harris WH \& Heaney RP. Effect of growth hormone on skeletal mass in adult dogs. Nature 1969;223:403-4.

43. Rudman D, Feller AG, Nagraj HS, Gergans GA, Lalitha PY, Goldberg AF, Schlenker RA, Cohn L, Rudman IW \& Mattson DE. Effects of human growth hormone in men over 60 years old. New England Journal of Medicine 1990;323:1-6.

44. Brixen, K, Kassem, M, Eriksen, E.F, Nielsen, H.K, Flyvbjerg, A. \& Mosekilde, L. Growth hormone (GH) and adult bone remodeling: the potential use of $\mathrm{GH}$ in treatment of osteoporosis. Journal of Pediatric Endocrinology \& Metabolism 1993;6:65-71.

45. Bak B. \& Andreassen T.T. The effect of growth hormone on fracture healing in old rats. Bone 1991;12:151-4.
46. Guicheux J, Gauthier O, Aguado E, Pilet P, Couillaud S, Jegou D, Daculsi, G. \& Heymann D. Human growth hormone locally released in bone sites by calciumphosphate biomaterial stimulates ceramic bone substitution without systemic effects: a rabbit study. Journal of Bone \& Mineral Research 1998;13:739-48.

\section{CORRESPONDENCIA}

Prof. A. Cutando

Facultad de Odontología.

Universidad de Granada.

Campus Universitario de Cartuja, $\mathrm{s} / \mathrm{n}$

18071 Granada, Spain.

Telf.: +34-958249653; E-mail: acutando@ugr.es 